\title{
BMJ Open Application of two versions of the WHO/international society of hypertension absolute cardiovascular risk assessment tools in a rural Bangladeshi population
}

\author{
Kaniz Fatema, ${ }^{1,2}$ Nicholas Arnold Zwar, ${ }^{2}$ Abul Hasnat Milton, ${ }^{3}$ Bayzidur Rahman, ${ }^{2}$ \\ Liaquat $\mathrm{Ali}^{4}$
}

To cite: Fatema K, Zwar NA, Milton AH, et al. Application of two versions of the $\mathrm{WHO} /$ international society of hypertension absolute cardiovascular risk assessment tools in a rural Bangladeshi population. BMJ Open 2015;5:e008140. doi:10.1136/bmjopen-2015008140

- Prepublication history for this paper is available online. To view these files please visit the journal online (http://dx.doi.org/10.1136/ bmjopen-2015-008140).

Received 11 March 2015 Revised 6 August 2015 Accepted 12 August 2015

CrossMark

For numbered affiliations see end of article.

Correspondence to

Dr Kaniz Fatema;

k.fatema@student.unsw.edu. $\mathrm{au}$

\section{ABSTRACT}

Objectives: To estimate the absolute cardiovascular disease (CVD) risk burden in a remote rural Bangladeshi population using the 'With' and 'Without' Cholesterol versions of the WHO/International Society of Hypertension (WHO/SH) CVD risk assessment chart (particularly suitable for low and middle-income countries due to less reliance on laboratory testing) and to evaluate the agreement between the two approaches.

Design: Cross-sectional study using data from a large prospective cohort of the North Bengal NonCommunicable Disease Programme (NB-NCDP) of Bangladesh.

Setting: General rural population from Thakurgaon district of Bangladesh.

Participants: 563 individuals who were categorised as having 'no CVDs' on screening by a questionnairebased survey using the 'WHO CVD-Risk Management Package' developed in 2002.

Main outcome measures: Absolute CVD risk burden assessed using two versions of the WHO/ISH risk assessment charts for the South-East Asian Region-D.

Results: 10-year risk (moderate, high and very high) positivity was present among $21.5 \%$ and $20.2 \%$ of participants, respectively, using with and without cholesterol versions of the tool. The overall concordance rate for the two versions was $89.5 \%$ and they did not differ significantly in estimating the proportion of overall participants having higher levels of CVD. The projected drug requirement, however, showed a significant overestimation in the proportion of participants at both the threshold levels $(p<0.002)$ on using 'without' as compared to 'with' cholesterol versions.

Conclusions: About one-fifth of the adult population in Bangladesh, even in a remote rural area, seem to be at risk of developing CVDs (25\% of them at high risk and $25 \%$ at very high risk) within 10 years with males and females being almost equally vulnerable.

\section{Strengths and limitations of this study}

- Community-based study in a remote location on a relatively traditional rural population; the second reported work on absolute cardiovascular disease (CVD) risk assessment on any Bangladeshi population.

- Both without and with cholesterol versions of the WHO/International Society of Hypertension tool were used in parallel, for the first time in a CVD-related study on a Bangladeshi population; generates baseline data for future communitybased cohort studies.

- The sample size is relatively small, limiting detailed analysis among various subgroups; the cross-sectional nature of the study does not allow inferences to be drawn on trends and causality.

- Comparison with other absolute risk assessment tools (like Framingham scoring) was not carried out.

\section{INTRODUCTION}

Cardiovascular diseases (CVDs) are now the major cause of death all over the world. It is estimated by the WHO that 17.5 million (31\% of all death) people died from CVDs in $2012 .{ }^{1}$ Of all deaths, about $80 \%$ occurred in low-income and middle-income countries (LMICs). ${ }^{2}$ Accurate estimation of CVD risk is vital for both prevention and management of CVDs as well as for designing long-term policies and programmes to combat the challenges posed by them. It is now known that many of the risk factors such as smoking, hypertension, dyslipidaemia, diabetes, physical inactivity and obesity are potentially modifiable by health counselling. ${ }^{3}$ Available evidence suggests that absolute CVD risk assessment on the basis of the combined 
effect of multiple risk factors yields a greater degree of accuracy than estimates based on individual risk factors. ${ }^{45}$ Moreover, existing evidence also indicates that the cost-effectiveness of management and treatment decisions, including the use of medicine, should be based on absolute CVD risk assessment. ${ }^{6}$

Most of the existing CVD risk-profiling packages rely on equations based on Framingham and other similar studies (eg, PROCAM (Munster), Seven Countries Study, SCORE and Progetto CUORE studies).$^{7-9}$ There is limited evidence of the applicability of these protocols in the context of developing countries, whose populations were not included in any of the aforementioned study samples. In 2000, after consultation with experts in all the WHO regions, the WHO released a risk-profiling tool tailored to developing countries that aimed to facilitate systematic case management at all healthcare levels. In 2007, this tool provided the framework for sets of regional risk prediction charts developed by the WHO and the International Society of Hypertension (WHO/ ISH). These were based on risk factors that could be assessed by physician and non-physician health workers in a primary care setting in each of the 14 WHO subregions. ${ }^{10}$ In recognition of resource limitations of LMICs, these tools placed a low emphasis on laboratory investigations ${ }^{11}$ and aimed to guide the allocation of limited healthcare resources in the most cost-effective way among high-risk groups. ${ }^{10}$ Although the CVD risk scores were originally intended for a quick, consistent measure of absolute risk in individuals, through cross-sectional surveys of population samples, they could also provide a tool for estimating and monitoring population trends of CVD risks. ${ }^{12} 13$

Bangladesh is an LMIC where the emerging challenge in the health sector is non-communicable diseases (NCDs) and, among all NCDs, the foremost cause of death and disability is CVDs. ${ }^{2} 14$ Approximately $13.4 \%$ of disability adjusted life years (DALYs) lost in Bangladesh are due to CVDs. ${ }^{15}$ These problems are exacerbated, especially in primary healthcare sectors, by socioeconomic obstacles, non-uniform accessibility to treatment, insufficient staffing of healthcare facilities and limited capacity for ancillary investigations.

Few LMICs currently have clinical guidelines either for screening or treatment of risk factors based on 'absolute' CVD risk scores, or any sort of estimate on the population distribution of CVD risks over time. In Bangladesh, studies aimed at risk estimation for CVDs are still limited in number and even those which have been conducted have mostly used an individual rather than multiple risk factor approach. Cravedi $e t a l^{16}$ have conducted the only such study among urban and rural populations. However the rural population in this study was recruited from regions close to urbanised areas, so it may not represent the risk factor pattern in more remote rural populations. The present study was undertaken to assess the distribution of absolute CVD risk in a remote rural $^{i}$ adult population, with and without cholesterol, using the $\mathrm{WHO} / \mathrm{ISH}$ risk prediction charts and to compare the concordance between the two approaches with the tool including cholesterol treated as the gold standard. We also compared the projected drug requirements for CVD prevention based on each version of the assessment tool at risk thresholds of $\geq 20 \%$ and $\geq 30$ for single risk factors in order to assess applicability of the tool in an LMIC setting.

\section{METHODS}

The selection of participants for the study has been described previously. ${ }^{17}$ Briefly, a prospective cohort of (190 471) residents in a rural area of Bangladesh was established in 2008 as part of the 'BADAS-ORBIS Eye Care Project'. The main objective was designed to generate epidemiological data on the burden of diabetic retinopathy and associated risk factors. In 2011-2012, participants in the eye project cohort who had been aged between 31 and 74 in 2008 were invited to participate in the North Bengal Non-Communicable Disease Programme (NB-NCDP) of Bangladesh University of Health Sciences (BUHS). Of 66701 eligible residents, 63708 consented (participation rate 95.5\%) and were screened for CVDs using the 'WHO CVD-RISK Management Package for low and medium resource settings'. ${ }^{11}$ This questionnaire identified individuals at high risk of a CVD event on the basis of current symptoms or a history of pre-existing CVD. The screening process identified 1170 individuals as high risk,${ }^{11}$ who were then immediately referred to the next level care, following the WHO package guidelines. Among the 62538 remaining cohort members, 1000 participants were randomly selected with the expectation that in the future, after a $50 \%$ loss to follow-up, we would have sufficient participants to observe an association, as per a case cohort design. Of the 1000 people invited, 563 (56.3\%) were willing to participate. The participants of this subcohort were checked for age, sex and education status with the main cohort and did not observe any significant difference between participants and non-participants.

Using a structured, pretested interviewer-administrated questionnaire, information was collected on the following: sociodemographic and economic characteristics, a 3-day dietary intake history (including fruit and vegetable intake), current tobacco use (including type of smoking or non-smoked tobacco use), smoking history, passive smoking, alcohol intake (including local form of alcoholic beverages), physical activity (assessed by exact daily duration (minutes) of work-related, commute-related and leisure time-related physical activity) and history of medicine intake for any chronic disease. A health examination collected anthropometric

${ }^{\mathrm{i}}$ Most of the existing research conducted in Bangladeshi rural settings has been in rural areas adjacent to urban spaces, whereas a remote rural area is one geographically and behaviourally distinct from urban influences 
measurements (ie, height, weight, waist and hip circumferences), blood pressure measurements $((B P)$, measured 3 times in sitting position), fasting and $2 \mathrm{hBG}(2 \mathrm{~h}$ blood glucose after having $75 \mathrm{~g}$ oral glucose for the oral glucose tolerance test) and blood sample collection for biochemical measurements (ie, lipid profile). Whole capillary blood was taken and centrifuged serum was separated and refrigerated for analysis. Plasma glucose was measured by the glucose oxidase method using DimalesionRxL Max (Siemens AG, Erlangen, Germany) and plasma-TC was analysed by the enzymatic colorimetric method. Verbal consent in the presence of a witness was obtained for all participants.

\section{Calculating absolute CVD risk using the WHO/ISH risk prediction charts}

To classify absolute CVD risk, individuals were evaluated using the WHO/ISH risk assessment charts for the South-East Asian Region: D. The WHO and ISH have devised two sets of risk prediction charts-with and without blood cholesterol. ${ }^{11}{ }^{18}$ Both sets require data on sex (male/female), age in years $(31-49,50-59,60-69$ and $\geq 70$ years $)$, systolic blood pressure $(<140 \mathrm{~mm} \mathrm{Hg} ; 140$ $160 \mathrm{~mm} \mathrm{Hg}, 160-180 \mathrm{~mm} \mathrm{Hg}$ and $\geq 180 \mathrm{~mm} \mathrm{Hg}$, the mean of the last two of the three measurements taken in rested participants in sitting position), smoking status (no, yes, ex-smoker $<12$ months), presence or absence of diabetes status (yes/no, defined by fasting blood glucose $\geq 7 \mathrm{mmol} / \mathrm{L}$ ) and record of oral hypoglycaemic drug use if blood sugar is measured below these threshold levels, in order to estimate risk of a cardiovascular event in the next 10 years. The chart developed for settings where blood cholesterol can be measured also uses blood cholesterol (total cholesterol in five categories: $<5 \mathrm{mmol} / \mathrm{L}, 5-<6 \mathrm{mmol} / \mathrm{L}$, $6-<7 \mathrm{mmol} / \mathrm{L}, 7-<8 \mathrm{mmol} / \mathrm{L}$ and $\geq 8 \mathrm{mmol} / \mathrm{L})$. The charts provide evidence-based recommendations on the initiation of specific preventive actions (behavioural or pharmacological) and their intensity. In this study, we categorised the population according to the risk of developing a fatal or non-fatal severe CVD event (acute myocardial infarction and/or stroke) in the next 10 years into four levels: low, $<10 \%$; moderate, 10-19.9\%; high, $20-29.9 \%$; and very high risk, $\geq 30 \% .^{18} 19$ The WHO criteria and recommendations as well as expert consensus recommend drug therapy to prevent CVD in people with a CVD risk threshold $\geq 30 \%$, or $\geq 20 \%$ if financially feasible. ${ }^{10} 19-21$

\section{Statistical methods}

Analysis was conducted using Stata V.12 (StataCorp LP, College Station, Texas, USA). Percentages with 95\% CIs were calculated for categorical variables. Means, SDs and 95\% CIs were calculated for continuous variables. Excel file 2003 (Microsoft) was used for CVD risk calculation, data entry and processing. For CVD risk prediction analysis, we used the method described in the WHO guidelines. ${ }^{10}$ We used the $\chi^{2}$ test for statistical significance with a threshold of $p<0.05$. For data analysis, we used the method described in the WHO guidelines. $^{22}$ Concordance between the two prediction charts was calculated for the different risk levels assuming the chart with cholesterol as the reference (ie, if CVD risk was classified higher without cholesterol, we described it as an overestimate; if lower, as an underestimate). Cohen's $\kappa$ was used to observe the inter-rater agreement between with and without cholesterol for a 10 year CVD risk score.

\section{RESULTS}

Overview of the risk burden among study participants After analysis, the overall demographic and social characteristics of all the study participants showed that male representation was higher $(66.1 \%)$, about threequarters of the participants $(73 \%)$ had low or poor educational knowledge (ie, illiterate/only signature/ gonoshikha and primary education) and almost 99\% were from the low and lower middle-income group and $67.1 \%$ of them were from the physically active group (ie, homemaker /farmer) (data not shown on a table).

\section{Distribution of individual risk factors used in absolute CVD-risk assessment}

Among the 563 participants (aged 31-74 years), mean ages were $51.9 \pm 10.6$ versus $47.7 \pm 10.8$ years for males versus females, respectively. Among GVD risk factors, prevalence of smoking was the highest $(75.8 \%)$, followed by systolic blood pressure $\geq 140 \mathrm{~mm} \mathrm{Hg}(9.1 \%)$, fasting blood glucose $\geq 7 \mathrm{mmol} / \mathrm{L}(2.7 \%)$ and fasting blood cholesterol $\geq 6 \mathrm{mmol} / \mathrm{L}(18.1 \%)$. The trends by age and sex should be noted as the WHO/ISH charts include both of these demographic characteristics as the key defining variables in the risk charts (table 1).

\section{Distribution of 'absolute' CVD risk using the WHO/ISH risk prediction charts alone}

The distribution of absolute CVD risk using both sets (with and without cholesterol) is shown in table 2. When risk assessment was performed with cholesterol, $89 \%$ had a 10 -year CVD risk of $<20 \%$; the corresponding proportion was $92.2 \%$ when cholesterol was not included in the calculation. The proportions with risk $\geq 20 \%$ (high and very high) were $11 \%$ with cholesterol versus $7.8 \%$ without. When we applied the charts without cholesterol, the percentage of the population in the high (20-29.9\% 10-year risk) and very high-risk categories ( $\geq 30 \%$ 10-year risk) was less than with the chart using cholesterol (4.3 vs 5.3 and 3.6 vs 5.7 , respectively), but the difference was not statistically significant. In general, males had a higher risk of CVD according to both the tools.

\section{Concordance of the two CVD risk charts}

In this study, concordance was found between the two charts for $504(89.5 \%)$ participants (table 3). Using the with cholesterol version as the gold standard, the chart 
Table 1 Gender and age distribution and the prevalence of CVD risk factors of the study participants $(n=563)$

\begin{tabular}{|c|c|c|c|c|c|c|}
\hline & \multicolumn{6}{|l|}{ Age group (years) } \\
\hline & $31-49$ & $50-59$ & $60-69$ & $\geq 70$ & Total n (\%) & Mean \pm SD \\
\hline \multicolumn{7}{|l|}{ Gender } \\
\hline Male & $152(40.9)$ & $116(31.2)$ & 74 (19.9) & $30(8.1)$ & $372(66.1)$ & $51.9 \pm 10.6$ \\
\hline Female & $110(57.6)$ & 47 (24.6) & $23(12.0)$ & $11(5.8)$ & 191 (33.9) & $47.7 \pm 10.8$ \\
\hline Total n (\%) & $262(46.5)$ & $163(29.0)$ & 97 (17.2) & 41 (7.3) & $563(100)$ & $50.5 \pm 10.9$ \\
\hline Variables & \multicolumn{6}{|c|}{ Prevalence of CVD risk factors \% $(95 \% \mathrm{Cl})$} \\
\hline${ }^{*} \mathrm{HTN}$ & 5.3 (3.0 to 8.8$)$ & 6.7 (3.4 to 11.8$)$ & 20.6 (13.1 to 30.0$)$ & 14.6 (5.6 to 29.2$)$ & $9.1(6.8$ to 11.7$)$ & - \\
\hline †DM & $2.0(0.6$ to 4.4$)$ & $1.8(0.4$ to 5.3$)$ & $5.2(1.7$ to 11.6$)$ & 2.7 (0.6 to 12.9$)$ & $2.7(1.5$ to 4.3$)$ & - \\
\hline$\ddagger \mathrm{HC}$ & 15.3 (11.1 to 20.2$)$ & 22.7 (16.5 to 29.9$)$ & 22.7 (14.8 to 32.3$)$ & $7.3(1.5$ to 19.9$)$ & 18.1 (15.0 to 21.6$)$ & - \\
\hline Smoking & 68.7 (62.7 to 74.3$)$ & 79.8 (72.8 to 85.6$)$ & 84.5 (75.8 to 91.1$)$ & 85.4 (70.8 to 94.4$)$ & 75.8 (72.1 to 79.3$)$ & - \\
\hline
\end{tabular}

without cholesterol overestimated CVD risk in $43(7.6 \%)$ participants and underestimated risk in $16(2.8 \%)$ participants. Of the 59 individuals with non-concordant CVD risk estimates, $72.8 \%(43 / 59)$ were overestimated (29/41 men and 14/18 women); $40.7 \%$ (24/59) belonged to low and moderate CVD risk categories that would not have drug intervention at a CVD threshold risk of $\geq 20 \%$ with either chart. Only $4.3 \%(24 / 563)$ of the total sample was misclassified vice versa need for drug intervention: risk was overestimated in $3.4 \%(19 / 563)$ and underestimated in $1.1 \%(6 / 563)$.

\section{Comparison of drug requirement among study participants}

Using the WHO guidelines for CVD risk assessment and management, (2) estimates of proportions of persons requiring medications to reduce CVD risk in this sample were similar by both with and without cholesterol charts. Using a threshold of $\geq 30 \% 10$-year risk, $6.1 \%$ of those studied without measuring cholesterol were classified as requiring drugs, $4.1 \%$ of those studied were those in whom cholesterol was measured. Using a threshold of $\geq 20 \%$, the proportions were $11.4 \%$ and $8.4 \%$, respectively. Applying the single-risk-factor approach, about $28.2 \%$ (159 of 563) of the participants would require drug treatment $(10.1 \%$ antihypertensives and $18.1 \%$ lipid-lowering drugs); almost fivefold higher than without cholesterol and sevenfold higher than with the cholesterol risk approach (table 4).

\section{DISCUSSION}

Data from this study show that about $10 \%$ of the adult populations, even in a peripherally located rural population of Bangladesh, are at high risk of a CVD event in the next 10 years and half of these individuals are in the very high-risk category. These proportions are similar whether the 'with or without' cholesterol version of the WHO/ISH CVD Risk-Assessment Tool is used. The risk is in terms of a 10-year absolute CVD Risk, which is much higher than that recently reported by Cravedi et $a l,{ }^{16}$ using only the without cholesterol version of the tool, in another rural Bangladeshi population. In that study, the proportion of higher risk participants is only $2.1 \%$ (very high risk $1.2 \%$ and high risk $0.9 \%$ ), whereas in this study, using the same tool, the corresponding proportion is $7.9 \%$ (very high risk $3.6 \%$ and high risk $4.3 \%)$. The reasons for these discrepancies are not presently known; however, population differences related to

Table 2 Ten-year absolute CVD risk prediction by gender, using the WHO/ISH SAER D charts, with and without cholesterol

\section{WHO/ISH SAER D chart}

With cholesterol

Without cholesterol

${ }^{*} \chi^{2}=0.35 \mathrm{p}=0.557$

$+\chi^{2}=0.88 p=0.348$

$\neq \chi^{2}=0.70 \mathrm{p}=0.403$

$\S \chi^{2}=2.90 p<0.088$.

CVD, cardiovascular disease, WHO/ISH, WHO/International Society of Hypertension.

\section{0-Year WHO/ISH cardiovascular risk prediction}

Male $77.7(289 / 372)$

Female $80.1(153 / 191$

Total $\quad 78.5(442 / 563)^{*} \quad 10.5(59 / 563) \dagger$

Male $\quad 78.5(292 / 372) \quad 13.2(49 / 372)$

Female $82.7(158 / 191) \quad 10.5(20 / 191)$

Total $79.9(450 / 563)^{*} \quad 12.3(69 / 563) \dagger$

Gender Low risk (<10) Moderate risk (10-19.9) High risk (20-29.9) Very high risk (30-39.9)

$\begin{array}{ll}5.9(22 / 372) & 5.1(19 / 372) \\ 4.2(8 / 191) & 6.8(13 / 191) \\ 5.3(30 / 563) \ddagger & 5.7(32 / 563)^{\S} \\ 5.6(21 / 372) & 2.7(10 / 372) \\ 1.6(3 / 191) & 5.2(10 / 191) \\ 4.3(24 / 563) \ddagger & 3.6(20 / 563)^{\S}\end{array}$


Table 3 Concordance of the WHO/ISH SAER D CVD risk charts with and without cholesterol for participants

\begin{tabular}{lllrrr}
\hline \multirow{2}{*}{$\begin{array}{l}\text { WV risk } \\
\text { With cholesterol }\end{array}$} & \multicolumn{6}{l}{ Without cholesterol N (\%) for participants } & \\
\cline { 2 - 6 } & Low risk (<10) & Moderate risk (10-19.9) & High risk (20-29.9) & Very high risk (30-39.9) & Total (\%) \\
\hline Low risk & $432(76.7)$ & $12(2.1)$ & $5(0.9)$ & $1(0.2)$ & $7(1.2)$ \\
Moderate risk & $10(1.8)$ & $41(7.3)$ & $11(2.0)$ & $7(1.2)$ & $450(79.9)$ \\
High risk & 0 & $3(0.5)$ & $14(2.5)$ & $17(3.0)$ & $24(4.3)$ \\
Very high risk & 0 & $3(0.5)$ & 0 & $32(5.7)$ & $20(3.6)$ \\
Total (\%) & $442(78.5)$ & $59(10.5)$ & $30(5.3)$ & & $563(100.0)$ \\
\hline
\end{tabular}

Concordance: $89.5 \%(504 / 563)$.

Non-concordance: $10.5 \%(59 / 563)$.

Overestimate: $7.6 \%(43 / 563)$.

Underestimate: $2.8 \%(16 / 563)$.

Misclassification for CVD risk threshold $\geq 20 \%: 4.3 \%$ (24/563).

Overestimate: $3.4 \%(19 / 563)$.

Underestimate: $1.1 \%(6 / 563)$.

$\kappa$ Value $(0.705, p<0.001)$

CVD, cardiovascular disease, WHO/ISH, WHO/International Society of Hypertension.

genetic as well as environmental, sociodemographic and lifestyle factors may contribute to the wide difference in the prevalence of the higher risk of CVDs among the two groups. It needs to be noted that the proportion of the 10-year absolute CVD risk positive (higher than the low risk group including the moderate CVD risk group) participants (25\% in the Cravedi study vs $20.2 \%$ in this study) are fairly similar in the two studies.

With the exception of the work as cited above, no other study has reported absolute CVD risk among the Bangladeshi population (using the WHO/ISH Tool or any other absolute risk scoring system). The without cholesterol version of the tool was applied in various other countries; a similar proportion of risk positive (moderate, high and very high) groups has been reported from Bolivia (11.3\%) and higher proportions have been reported from Nepal (16.9\%), the USA
$(18.2 \%)$ and Georgia (25\%). ${ }^{12}$ Comparative data using 'with cholesterol' shows considerable similarity with our findings $(78.5 \%)$ for low risk $(<10 \%)$ group in Pakistan, Georgia, Nepal, Nigeria, Mongolia and Cuba (79.2\%, $83.1 \%, 87.2 \%, 86 \%, 89.6 \%$ and $89.7 \%$ respectively); and somewhat $10 \%$ higher in Iran, Sri Lanka, Malaysia, China and Cambodia $(93.9 \%, 94.9 \%, 94.4 \%, 96.1 \%$ and $97 \%$, respectively). However, among the moderate or high-risk groups, males show a higher preponderance in almost all countries except Sri Lanka and Nepal, which is similar to our findings. ${ }^{12} 2324$

The major focus of this study was to find out the level of agreement between the results generated by the without and with cholesterol versions of the WHO/ISH Tool. From the frequency distribution (table 2), it can be seen that the results from the two versions do not differ significantly when both genders are considered as a

Table 4 Association of drug requirements for CVD prevention among the study population as per total CVD risk assessment, with and without cholesterol, and single risk factor line

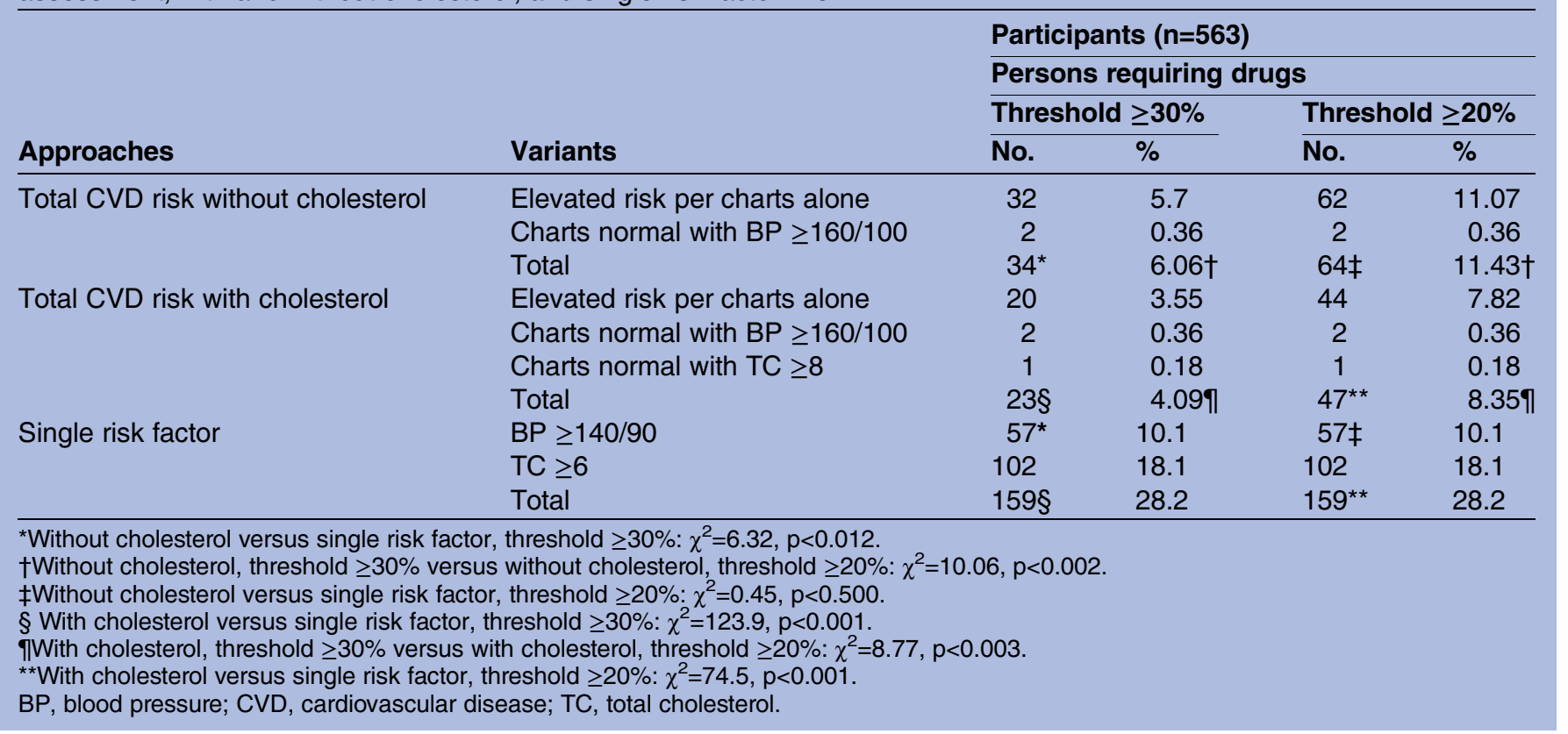


combined group. However, when males and females are analysed separately, wide discrepancies are found in the results of the two charts. There is a gross chance of overestimation (by about $71 \%$ ) of very high risk among males and (by about $78 \%$ ) of high risk among females in using without cholesterol compared with the cholesterol version. Similarly, when the concordance between the two versions was statistically tested, the agreement between the two versions was fair $(89.5 \%)$ considering the subject group as a whole. However, repeating the tests separately in males and females resulted in similar levels of concordance $(89 \%$ and $90.6 \%$, respectively). On the basis of the overall population, the concordance rate in this study is similar to that in a Cuban study, ${ }^{23}$ but it is higher than that in a study which applied a Framingham CVD risk score using cholesterol and/or BMI formulae. ${ }^{25}$

In this study, the agreement was further explored by an empirical technique using the need for medication as suggested by the two versions of the WHO/ISH Tool. It can be seen that, compared to the single factor risk assessment approach, a much less proportion of participants require drug treatment when they are scored by the WHO/ISH Tool. However, this percentage of the population is about fivefold to sevenfold higher (whether the threshold of the absolute risk is $>30 \%$ or $>20 \%$ ) when suggestion is generated by the with cholesterol version compared to the without cholesterol version of the Tool. It thus appears that the reduction of the CVD risk threshold from $\geq 30 \%$ to $\geq 20 \%$ greatly improves the diagnosis of patients and did not introduce overprescription of drugs. However, both tools assessed better targeting of those more likely to develop CVD and, compare with the single-risk-factor approach, still showed either an underestimate or less need for drugs in a substantial proportion of participants. There is still no universal consensus on the threshold level $(>30 \%$ vs $20 \%$ ) that is required to make a decision for drug treatment ${ }^{1223}$ and the question may still be raised whether the absolute risk factor approaches may introduce undertreatment. The WHO proposes that the GVD risk threshold for intensive intervention across a 10-year span should be based on the resource levels of respective countries. ${ }^{23}$ Moreover, with or without cholesterol guidelines are not compared to any gold standard, that is, the WHO risk score (for region) is based on the modelling of various data from different countries but not on real data in Bangladesh (ie, there are no cohort studies in Bangladesh or neighbouring countries providing actual prospective CVD outcome data in relation to risk factors in these countries). Therefore, it is obvious that much of the data, particularly those based on longitudinal studies, need to be generated in Bangladesh to initiate more evidence-based discussion in this field.

In conclusion, about one-fifth of the adult people in Bangladesh, even in a remote rural area, seem to be at risk of developing CVDs within 10 years. Among this, one-fifth, about $25 \%$, are at high risk and another $25 \%$ are at very high risk of developing the disorders with males and females being almost equally vulnerable. Our data indicate that use of the with cholesterol version of the WHO/ISH 10-year absolute CVDs risk assessment could avoid overestimation of the risk, particularly in the high-risk and very high-risk subgroups, and undertreatment of participants who should be treated with medication. On the other hand, a very high concordance between the with and without cholesterol tools indicates that with the resource limitations and less facilities for laboratory investigations settings, the without cholesterol tools allow for better targeting of those who are more likely to develop cardiovascular diseases. The finding also indicates that a large-scale study could be conducted to verify the pattern.

\section{Author affiliations}

${ }^{1}$ Department of Epidemiology, Bangladesh University of Health Sciences (BUHS), Dhaka, Bangladesh

${ }^{2}$ Faculty of Medicine, The School of Public Health and Community Medicine, The University of New South Wales, Sydney, New South Wales, Australia

${ }^{3}$ Faculty of Health and Medicine, Centre for Clinical Epidemiology and Biostatistics (CCEB), The School of Medicine and Public Health, The University of Newcastle, Newcastle, New South Wales, Australia

${ }^{4}$ Department of Biochemistry and Cell Biology, BUHS, Dhaka, Bangladesh

Acknowledgements The authors are indebted to all participants in this study. They also acknowledge the important contribution of the field workers, the Thakurgaon Swasthoseba Hospital and ORBIS International to make this work possible.

Contributors KF, NAZ and LA developed the concept. KF oversaw all aspects of the study design, field training, collection and assembly of data, statistical expertise and interpretation of analysis, and drafted the manuscript. KF, NAZ and LA provided a critical revision of the article for important intellectual content. All authors read and approved the final manuscript.

Funding This work was supported by grants from the Bangladesh University of Health Sciences (BUHS).

Competing interests None declared.

Patient consent Obtained.

Ethics approval Human Research Ethical Committee (HREC) of the University of New South Wales (HREC ref: $\neq$ HC12621), Sydney, Australia and the Ethics Review Committee of the Diabetic Association of Bangladesh (DAB).

Provenance and peer review Not commissioned; externally peer reviewed.

Data sharing statement No additional data are available.

Open Access This is an Open Access article distributed in accordance with the Creative Commons Attribution Non Commercial (CC BY-NC 4.0) license, which permits others to distribute, remix, adapt, build upon this work noncommercially, and license their derivative works on different terms, provided the original work is properly cited and the use is non-commercial. See: http:// creativecommons.org/licenses/by-nc/4.0/

\section{REFERENCES}

1. World Health Organization (WHO). Cardiovascular Diseases (CVDs). Fact Sheet N 317. January. 2015. http://www.who.int/ mediacentre/factsheets/fs317/en/index.html (updated January, 2015 and accessed 5 Jan 2015)

2. Mendis S, Puska P, Norrving B. Global atlas on cardiovascular disease prevention and control. World Health Organization, 2011.

3. Hussain SM, Oldenburg B, Wang Y, et al. Assessment of cardiovascular disease risk in south Asian populations. Int $J$ Vasc Med 2013;2013:786801. 
4. Jackson R, Lawes CM, Bennett DA, et al. Treatment with drugs to lower blood pressure and blood cholesterol based on an individual's absolute cardiovascular risk. Lancet 2005;365:434-41.

5. Kannel WB, McGee D. Diabetes and glucose tolerance as risk factors for cardiovascular disease: the Framingham study. Diabetes Care 1979;2:120-6.

6. Murray CJ, Lauer JA, Hutubessy RC, et al. Effectiveness and costs of interventions to lower systolic blood pressure and cholesterol: a global and regional analysis on reduction of cardiovascular-disease risk. Lancet 2003;361:717-25.

7. Assmann G, Cullen P, Schulte H. Simple scoring scheme for calculating the risk of acute coronary events based on the 10-year follow-up of the prospective cardiovascular Münster (PROCAM) study. Circulation 2002;105:310-15.

8. Conroy RM, Pyörälä K, Fitzgerald AP, et al., SCORE project group. Estimation of ten-year risk of fatal cardiovascular disease in Europe: the SCORE project. Eur Heart $J$ 2003;24:987-1003.

9. Ferrario M, Chiodini P, Chambless LE, et al., CUORE Project Research Group. Prediction of coronary events in a low incidence population. Assessing accuracy of the CUORE Cohort Study prediction equation. Int J Epidemiol 2005;34:413-21.

10. World Health Organization (WHO). Prevention of cardiovascular disease: Guidelines for assessment and management of cardiovascular risk. 2007:86. http://whqlibdoc.who.int/publications/ 2007/9789241547178_eng.pdf?ua=1 [published Online First: Epub Date].

11. World Health Organization (WHO). Cardiovascular disease programme. WHO CVD-risk management package for low-and medium-resource settings. Geneva, Switzerland: World Health Organization, 2002.

12. Mendis S, Lindholm LH, Anderson SG, et al. Total cardiovascular risk approach to improve efficiency of cardiovascular prevention in resource constrain settings. J Clin Epidemiol 2011;64:1451-62.

13. Ndindjock R, Gedeon J, Mendis S, et al. Potential impact of single-risk-factor versus total risk management for the prevention of cardiovascular events in Seychelles. Bull World Health Organ 2011:89:286-95.

14. Ghaffar A, Reddy KS, Singhi M. Burden of non-communicable diseases in South Asia. BMJ 2004;328:807-10.
15. El-Saharty S, Ahsan KZ, Koehlmoos TL, et al. Tackling noncommunicable diseases in Bangladesh: now is the time. Direstion in Development. Washington DC: World Bank Publications, 2013.

16. Cravedi P, Sharma SK, Bravo RF, et al. Preventing renal and cardiovascular risk by renal function assessment: insights from a cross-sectional study in low-income countries and the USA. BMJ Open 2012;2:pii: bmjopen-2012-001357.

17. Fatema K, Zwar NA, Zeba Z, et al. Clinical and biochemical characterization of high risk and not high risk for cardiovascular disease adults in a population from peripheral region of Bangladesh. BMC Public Health 2015;15:559.

18. Joint United Nations Programme on HIV/AIDS (UNAIDS) \& World Health Organigation (WHO). Prevention of cardiovascular disease. World Health Organization, 2007.

19. World Health Organization (WHO). Prevention of cardiovascular disease: pocket guidelines for assessment and management of cardiovascular risk:(WHO/ISH cardiovascular risk prediction charts for the European Region). Geneva, Switzerland: WHO Press, World Health Organization, 2007.

20. Williams B, Poulter NR, Brown MJ, et al., BHS guidelines working party, for the British Hypertension Society. British Hypertension Society guidelines for hypertension management 2004 (BHS-IV): summary. BMJ 2004;328:634-40.

21. Jackson R. Updated New Zealand cardiovascular disease risk-benefit prediction guide. BMJ 2000;320:709-10.

22. World Health Organization (WHO). Noncommunicable disease and poverty. The need for pro-poor strategies in the Western Pacific Region: Manila: World Health Organization, Western Pacific Region, 2006.

23. Nordet $\mathrm{P}$, Mendis $\mathrm{S}$, Dueñas A, et al. Total cardiovascular risk assessment and management using two prediction tools, with and without blood cholesterol. MEDICC Rev 2013;15:36-40.

24. Otgontuya D, Oum S, Buckley BS, et al. Assessment of total cardiovascular risk using WHO/ISH risk prediction charts in three low and middle income countries in Asia. BMC Public Health 2013;13:539.

25. Green BB, Anderson ML, Cook AJ, et al. Using body mass index data in the electronic health record to calculate cardiovascular risk. Am J Prev Med 2012;42:342-7. 